\title{
CATALOGAÇÃO DE JOGOS ELETRÔNICOS ${ }^{1}$ \\ CATALOGING OF ELECTRONIC GAMES
}

\author{
Jackson Salm \\ Graduado em Biblioteconomia \\ jacksonsalm@gmail.com \\ Araci Isaltina de Andrade Hillesheim \\ Mestre em Educação \\ Professora do DCI-UFSC \\ araci.h@ufsc.br
}

Recebido em: 11-04-2019

Aceito em: 05-06-2019

\begin{abstract}
Resumo: A pesquisa aborda a catalogação de jogos eletrônicos. Tem como objetivo analisar as normas definidas pelo Resource Description and Access (RDA) para catalogação de jogos eletrônicos. Apresenta algumas diferenças entre o Código de Catalogação Anglo-Americano $2^{a}$ edição (AACR2) e o RDA. Por meio de uma pesquisa bibliográfica, com caráter exploratório, lista e conceitua elementos considerados importantes para catalogar jogos eletrônicos. Faz uma releitura das regras de catalogação do RDA para jogos eletrônicos, apresentando-as de forma mais simples. Aplica as regras de catalogação para descrever alguns itens. Apresenta as principais dificuldades encontradas para a catalogação de jogos e sugere um maior aprofundamento sobre os conceitos envolvidos acerca do RDA e suas funcionalidades para a catalogação de jogos eletrônicos, bem como o desenvolvimento de pesquisas empíricas que demonstrem os problemas e vantagens do uso do RDA para a descrição de jogos eletrônicos.
\end{abstract}

Palavras-chave: Catalogação - Jogos eletrônicos. Resource Description and Access (RDA). Jogos eletrônicos.

\begin{abstract}
The research reports the cataloging of electronic games. Its objective is to analyze the norms defined by the Resource Description and Access (RDA) for the cataloging of electronic games. It presents some differences between the Anglo-American Cataloging Code 2nd edition (AACR2) and the RDA. Through a bibliographical research, with exploratory character, list and conceptualize elements considered important to catalog electronic games. Makes a rereading the RDA cataloging rules for electronic games, presenting them in a more simple form. Applies the cataloguing rules to describe some items. It presents the main difficulties encountered for the cataloging of games and suggests a deeper understanding of the concepts involved about the RDA and its functionalities for the cataloging of electronic games, as well as the development of empirical researches that demonstrate the problems and advantages of using the RDA for the description of electronic games.
\end{abstract}

Keywords: Cataloging - Electronic games. Resource Description and Access (RDA). Electronic games.

\section{INTRODUÇÃO}

As bibliotecas deixaram de ter somente recursos informacionais impressos. $\mathrm{O}$ avanço tecnológico proporcionou o crescimento de uma diversidade de recursos criados tanto no meio analógico quanto digital. Dentre essa variedade de recursos, destaca-se os jogos eletrônicos, foco desta pesquisa.

Desde o surgimento do primeiro jogo eletrônico, criado em 1958 por Willy Higinbotham, os jogos evoluíram consideravelmente. Os gráficos passaram de duas dimensões para três dimensões. Os suportes, com

\footnotetext{
${ }^{1}$ Artigo oriundo do Trabalho de Conclusão de Curso de Graduação em Biblioteconomia da Universidade Federal de Santa Catarina, em 2017.
} 
o desenvolvimento da tecnologia, mudaram de cartuchos para os CD-ROM. Atualmente é possível adquirir jogos por meio de plataformas digitais, via Internet, sem a necessidade de uma mídia física ou seu respectivo leitor, facilitando a sua execução em computadores e celulares (SANTOS JÚNIOR; NUNES, 2016).

Como os jogos eletrônicos vêm tomando espaço no cotidiano das pessoas, garantir o acesso a eles se torna cada vez mais importante. O acesso vai depender de como essas informações podem ser recuperadas. A efetividade da recuperação é um resultado direto da organização dessa informação. Fabricantes e consumidores precisam de formas padronizadas de organizar as coleções de jogos eletrônicos, a fim de assegurar/facilitar seu acesso.

A área da Biblioteconomia dispõe de diversas técnicas que visam a organização de coleções, tornando a informação acessível. Essas práticas são a catalogação, a classificação e a indexação. Nesta pesquisa destacase a catalogação, também conhecida como representação descritiva, que caracteriza-se pelo conjunto de dados que são determinados a partir da análise de um recurso informacional, fornecendo uma descrição única.

Observa-se que na prática, os jogos eletrônicos são classificados e indexados de acordo com seu gênero, personagem principal e/ou franquia, não fornecendo subsídios suficientes para garantir uma recuperação efetiva. Sendo assim, a catalogação, por fornecer uma descrição mais completa, complementa a representação desse tipo de material.

Perante o contexto apresentado e a natureza única dos jogos eletrônicos despertaram o interesse em buscar na literatura respostas para a seguintes perguntas: Como as regras de catalogação existentes orientam para a descrição de jogos eletrônicos? Quais os elementos descritivos para os jogos eletrônicos?

Diante disso, a pesquisa teve como principal objetivo analisar as normas definidas pelo Resource Description and Access (RDA) para catalogação de jogos eletrônicos. Para isso, foram identificados os elementos necessários para catalogar jogos eletrônicos, visando aplicar as regras estudadas.

\section{CATALOGAÇÃO EM RDA}

A catalogação é a descrição física e bibliográfica de um documento, a fim de torná-lo único no acervo de uma unidade de informação, permitindo sua recuperação. Para Mey e Silveira (2009, p. 7), a catalogação pode ser definida como:

O estudo, preparação e organização de mensagens, com base em registros do conhecimento, reais ou ciberespaciais, existentes ou passíveis de inclusão em um ou vários acervos, de forma a permitir a interseção entre as mensagens contidas nestes registros do conhecimento e as mensagens internas dos usuários.

O processo de catalogação permite a criação de catálogos que facilitam a recuperação dos documentos e a visualização de dados que caracterizam as obras. No entanto, para a elaboração desses catálogos é fundamental que o processo de catalogação possua regras “[...] que ofereçam o máximo de padronização e minimizem as interpretações individuais, procurando garantir a unicidade do item informacional representado e, ao mesmo tempo, sua universalidade" (SANTOS; CORRÊA, 2009, p. 19). 
Foram muitas as tentativas de elaboração de códigos que padronizassem o processo de catalogação. Entre tantas reivindicações, a segunda edição do Código de Catalogação Anglo-Americano (Anglo-American Cataloging Rules 2nd edition - AACR2), publicado em 1978, foi o primeiro código de catalogação aceito internacionalmente e amplamente utilizado no Brasil. Em 1988, a segunda edição revista do AACR2 foi publicada, sendo traduzida somente em 2004 no Brasil (MEY; SILVEIRA, 2009).

No entanto, em decorrência do avanço tecnológico e o desenvolvimento cada vez mais rápido da tecnologia de informação e comunicação, percebe-se a necessidade de repensar a forma de descrever os documentos nos diferentes formatos e de como registrar esta descrição, para uma recuperação precisa e eficaz nos sistemas automatizados de gerenciamento de unidades de informação.

Apesar da popularidade e funcionalidade reconhecida do AACR2 na área de organização e tratamento da informação, Baptista (2008, p. 2) afirma que:

[...] tem-se a clara consciência de que tais regras tornaram-se insuficientes para a descrição e recuperação de recursos típicos do ambiente da informação eletrônica, que nos dias de hoje extrapolam o ambiente e as práticas tradicionais de biblioteca, voltadas essas, em sua maior parte, para materiais impressos e cujas fontes de informação e/ou pontos de acesso se constituem em elementos previsíveis e estáticos, como é, por exemplo, o caso da folha de rosto, do colofão, de autor e título, etc.

Diante desta situação, foi criado o RDA, que apresenta novas regras para a descrição de recursos informacionais, desenvolvido para o ambiente digital (MACHADO; PEREIRA, 2017). Concebido pelo próprio Joint Steering Committee for Revision AACR (responsável pelo AACR) e apoiado pela International Federation of Library Associations and Institutions (IFLA), o novo código foi publicado, em 2010, desenvolvido com o intuito de substituir o AACR2.

O RDA é uma norma que oferece instruções de quais dados armazenar e como o fazer, afim de criar um grande grupo de informações que alimentam as bases de dados. Estes dados são analisados e distribuídos de modo a serem utilizados apropriadamente (SILVA et al., 2012).

Sua estrutura flexível é baseada nos modelos conceituais no Functional Requirements for Bibliographic Records (FRBR - Requisitos Funcionais para Registros Bibliográficos) e do Functional Requirements for Authority Data (FRAD - Requisitos Funcionais para Dados de Autoridade). "O modelo FRBR é o primeiro modelo conceitual definido para a catalogação, inspirado no modelo de entidaderelacionamento do mundo da informática. Assim, as entidades e seus atributos (características) e as relações entre eles são definidos" (PICCO, 2009, p. 153, tradução nossa). Já o FRAD é uma extensão do modelo FRBR para dados de autoridade. Ambos identificam e coletam os dados utilizados pelo usuário no processo de busca que formarão uma massa de informação essencial para a otimização dos recursos de todo o processo (OLIVER, 2011).

Embora algumas semelhanças permaneçam, na estrutura do RDA já nota-se uma das várias diferenças entre RDA e AACR2. Composto por 38 capítulos, começando do capítulo zero (introdução) ao capítulo 37, 13 
apêndices, glossário e índice. Os capítulos são dispostos em duas seções principais, divididas entre a parte A: Reccording Attributes (Registro de Atributos) e parte B: Recording Relationships (Registro de Relações), somando o total de 10 seções.

Assim, como a sua estrutura, os termos utilizados pelo AACR2 foram alterados no RDA. Os novos termos adotados pelo RDA foram introduzidos por refletirem melhor os conceitos e a terminologia dos modelos FRBR e FRAD. O termo resource (recurso) utilizado para nomear o código, por exemplo, foi adotado por expressar mais adequadamente todos os recursos que fazem parte do universo bibliográfico (TILLETT, 2007).

Segundo Oliver (2011), o RDA é voltado a construir registros que ajudam os usuários a encontrar um recurso particular. Evidencia-se isso pelo fato do uso de abreviaturas serem descartados no RDA. Enquanto o AACR2 utiliza abreviações com frequência, o RDA utiliza as informações por extenso, tornando o registro bibliográfico mais claro para o usuário. As abreviações são utilizadas no RDA em situações excepcionais como, por exemplo, quando a abreviatura é transcrita na fonte de informação ou para utilização de unidade de medida.

Outra diferença que o RDA apresenta em relação ao AACR2 é a regra dos três. Enquanto que no AACR2, quando há mais de três autores no mesmo nível de responsabilidade para uma obra, a regra estabelece que se deve omitir todos os nomes, exceto o do primeiro autor. No RDA, a indicação de responsabilidade é transcrita de acordo com o que consta na fonte de informação.

Quanto a pontuação, "o RDA não apresenta as pontuações estabelecidas como precedentes e sucedentes a cada área da descrição bibliográfica" (MEY; SILVEIRA, 2009, p. 126), como prescritas na International Standard Bibliographic Description (ISBD - Descrição Bibliográfica Padronizada Internacional). A ISBD representa um conjunto de elementos descritivos e a forma como se apresenta os dados bibliográficos, servindo como norma básica para a descrição bibliográfica em todos os países (OLIVER, 2011).

O código RDA é disponibilizado pela ferramenta on-line RDA Toolkit que oferece recursos e suporte ao catalogador, assim como atualizações e treinamentos para sua utilização. Para o acesso gratuito à ferramenta é possível fazer uma inscrição com duração de um mês.

\section{PROCEDIMENTOS METODOLÓGICOS}

A presente pesquisa caracteriza-se como bibliográfica por ser realizada a partir de referências teóricas publicadas e exploratória por ter como objetivo proporcionar maior familiaridade sobre determinado assunto (CERVO; BERVIAN; SILVA, 2007).

Dessa forma, a pesquisa foi realizada tendo como base artigos científicos indexados nas bases Web of Science (WoS), Scopus, Library and Information Science Abstracts (LISA) e Base de Dados Referenciais de Artigos de Periódicos em Ciência da Informação (BRAPCI). Para a recuperação dos documentos, optou-se por utilizar os termos catalogação, jogos eletrônicos e videogame escrito sob duas formas: videogame e vídeo 
game, presentes nos títulos, resumos ou nas palavras-chave dos artigos. Os termos foram utilizados em inglês, espanhol e português, combinados pelo operador booleano AND. Quanto ao tempo abrangido foi de sete anos (2010-2017), delimitado a partir do ano de 2010 por coincidir com o lançamento oficial da ferramenta RDA Toolkit na Internet. O resultado foi ordenado por relevância. Analisaram-se os resumos e as palavras-chave dos artigos que abordam especificamente o código RDA.

Foram recuperados artigos somente usando a combinação dos termos em inglês: catalogação e videogame escrito nas duas formas. Utilizando a combinação dos termos catalogação e videogame, obteve-se dois documentos recuperados na base WoS, três na Scopus e 13 na base LISA. Adotando os termos catalogação e vídeo game, não houve uma mudança expressiva nos resultados, apenas a WoS recuperou um artigo a mais dos encontrados, quanto as outras recuperaram o mesmo número de artigos. Não foi recuperado nenhum artigo na Brapci. Dos artigos recuperados, seis dissertavam a respeito de catalogação de jogos eletrônicos. Destes artigos, foram utilizados dois: Groat (2015) e Lee, Clarke e Perti (2015).

\section{REGRAS PARA DESCRIÇÃO DE JOGOS ELETRÔNICOS}

Os jogos eletrônicos são definidos por Aranha (2004) como dispositivos tecnológicos que utilizam imagens e áudio para formar um ambiente em televisões e monitores, para interagir com o seu jogador, nuca tiveram regras específicas para a sua descrição. A sua catalogação geralmente apontava regras mais gerais para materiais relacionados aos computadores.

Conforme Groat (2015), a primeira aparição de diretrizes para a catalogação desses recursos se deu com o capítulo 9 do AACR2, designado para descrição de recursos eletrônicos. Graças a alteração da regra 9.0B1 que passou a considerar a "tela de título" como fonte principal de informação, os jogos não são catalogados como se fossem artefatos tridimensionais ou realia, de acordo com o Capítulo 10 da AACR2.

As regras para a catalogação do RDA são semelhantes ao AACR2. A principal diferença entre AACR2 e RDA para a descrição de jogos é que a Designação Geral do Material (DGM) é omitida no RDA. Em vez disso, são registrados o tipo de conteúdo (RDA 6.9.1), tipo de mídia (RDA 3.2.1) e tipo de suporte (RDA 3.3.1).

O tipo de conteúdo é a forma de comunicação em que o conteúdo é expresso (RDA 6.9.1.1). Diz respeito também ao "[...] número de dimensões espaciais e a presença ou ausência de movimento em que o conteúdo expresso na forma de uma imagem ou imagens se destina a ser percebido" (RDA 6.9.1.1 alternativa). Nesse sentido, os jogos eletrônicos são considerados "imagem em movimento bidimensional" ou "imagem em movimento tridimensional". Segundo Groat (2015), alguns catalogadores também utilizam o termo "programa de computador" para definir o tipo de conteúdo.

O tipo de mídia refere-se a "categorização que reflete o tipo geral de dispositivo de intermediação necessário para visualizar, reproduzir, executar, etc., o conteúdo de uma manifestação" (RDA 3.2.1.1). Isso 
quer dizer, que tipo de equipamento é necessário para usar o recurso. No caso dos jogos eletrônicos, o tipo de mídia é um computador.

Já o suporte é definido como o formato do meio de armazenamento e a carcaça de uma manifestação (RDA 3.3.1.1). Entre os termos apontados pelo RDA para definir o suporte, os jogos eletrônicos podem ser: cartão para computador; cartucho para computador; disco para computador; recurso on-line. Se o termo não estiver na lista ou o catalogador preferir, poderá ser outro (RDA 3.3.1.3).

Segundo Lee, Clarke e Perti (2015), a Library of Congress descreve os jogos como se fossem imagens em movimento usando metadados projetados para filmes e gravações sonoras. No entanto, esta solução ainda é considerada insuficiente, pois não descrevem adequadamente os jogos de forma reconhecível para jogadores ou pesquisadores (OWENS, 2012).

Para resolver este problema, desde 2011, o Grupo GAme MEtadata Research (GAMER) da University of Washington Information School, em parceria com o Seattle Interactive Media Museum (SIMM), tem trabalhado na criação de um esquema de metadados padronizado e vocabulários controlados para jogos eletrônicos, representado pelo Quadro 1 (LEE; CLARKE; PERTI, 2015).

Quadro 1 - Elementos de metadados para descrição de jogos eletrônicos do Grupo GAMER

\begin{tabular}{|c|c|c|}
\hline Esquema & $\mathbf{N}^{\mathbf{0}} \mathbf{R D A}$ & Definiçãa \\
\hline Título $[\mathrm{O}][\mathrm{R}]$ & 5.1 .3 & $\begin{array}{l}\text { Nomes próprios que são usados para se referir a um jogo, atribuído } \\
\text { pelo criador. }\end{array}$ \\
\hline Edição & 2.5 .1 & $\begin{array}{l}\text { Uma palavra ou frase que aparece no jogo que está sendo descrito que } \\
\text { indica uma diferença no conteúdo ou forma entre ele e um jogo } \\
\text { relacionado (por exemplo, segunda edição, maiores sucessos, edição } \\
\text { de colecionador). }\end{array}$ \\
\hline Séries $[R]$ & 2.12 & $\begin{array}{l}\text { Nomes próprios de um conjunto de jogos relacionados, muitas vezes } \\
\text { indicados por numeração consecutiva, narrativa contínua, ou } \\
\text { semelhanças na jogabilidade e temas, aos quais o jogo que está sendo } \\
\text { descrito pertence. }\end{array}$ \\
\hline Franquias $[\mathrm{R}]$ & $\begin{array}{l}\text { Não há regra } \\
\text { associada }\end{array}$ & $\begin{array}{l}\text { Nomes comumente usados referentes à propriedade intelectual, dados } \\
\text { relacionados e conteúdo compartilhado entre um grupo de objetos } \\
\text { culturais aos quais o jogo que está sendo descrito pertence. }\end{array}$ \\
\hline $\begin{array}{l}\text { Entidade Coletiva } \\
{[\mathrm{O}][\mathrm{R}]}\end{array}$ & 11.2 & $\begin{array}{l}\text { Um indivíduo, organização ou grupo de indivíduos ou organizações } \\
\text { responsáveis pela criação, realização, fabricação, comercialização e/ou } \\
\text { distribuição de um jogo (modificado de FRBR, 2009, p.25). }\end{array}$ \\
\hline Plataforma $[\mathrm{O}][\mathrm{R}]$ & 3.2 & $\begin{array}{l}\text { O hardware e o sistema operacional no qual o jogo foi projetado para } \\
\text { ser reproduzido (por exemplo, PlayStation 4, Nintendo 3DS, Android } \\
\text { 7.0 Nougat, Windows 10). }\end{array}$ \\
\hline Formato [O] & 3.3 & $\begin{array}{l}\text { O meio ou método de distribuição que fornece o código executável de } \\
\text { um jogo eletrônico (por exemplo, cartucho, disco óptico). }\end{array}$ \\
\hline $\begin{array}{l}\text { Sistema requerido } \\
{[\mathrm{R}]}\end{array}$ & 3.20 & $\begin{array}{l}\text { Componentes de hardware, firmware e/ou software que são pré- } \\
\text { requisitos para a execução do jogo em uma plataforma específica (por } \\
\text { exemplo, } 2 \text { GB de RAM, } 8 \text { GB de espaço em disco). }\end{array}$ \\
\hline $\begin{array}{l}\text { Hardware } \\
\text { especial [R] }\end{array}$ & 3.20 & $\begin{array}{l}\text { Um hardware que é necessário ou recomendado para jogar o jogo para } \\
\text { além da plataforma principal (por exemplo, controlador de } \\
\text { movimento). }\end{array}$ \\
\hline $\begin{array}{l}\text { Recursos em rede } \\
{[\mathrm{R}]}\end{array}$ & $\begin{array}{l}\text { Não há regra } \\
\text { associada }\end{array}$ & $\begin{array}{l}\text { As formas pelas quais o jogo pode ser experimentado através de } \\
\text { ligações a outras entidades, tais como empresas de jogos, organizações }\end{array}$ \\
\hline
\end{tabular}




\begin{tabular}{|c|c|c|}
\hline & & $\begin{array}{l}\text { de terceiros e outros jogadores (por exemplo, multijogador on-line, } \\
\text { tabelas de líderes). }\end{array}$ \\
\hline Conectividade $[\mathrm{R}]$ & $\begin{array}{l}\text { Não há regra } \\
\text { associada }\end{array}$ & $\begin{array}{l}\text { A tecnologia por meio do qual os recursos de rede são realizados (por } \\
\text { exemplo, banda larga, LAN). }\end{array}$ \\
\hline $\begin{array}{l}\text { Conteúdo } \\
\text { adicional }[R]\end{array}$ & $\begin{array}{l}\text { J3.5; J4.5; J5.5 } \\
\quad \text { (varia } \\
\text { dependendo do } \\
\text { nível da FRBR) }\end{array}$ & $\begin{array}{l}\text { O tipo e o nome do conteúdo adicional, incluindo conteúdo para } \\
\text { download (DLC - Downloadable Content), modificações (Mod - } \\
\text { Modification), correções (Patches) e assim por diante. }\end{array}$ \\
\hline $\begin{array}{l}\text { Número de } \\
\text { jogadores }[\mathrm{O}][\mathrm{R}]\end{array}$ & $\begin{array}{l}\text { Não há regra } \\
\text { associada }\end{array}$ & $\begin{array}{l}\text { Número ou intervalo do número de jogadores que o jogo pode } \\
\text { acomodar separadamente ou concorrentemente. }\end{array}$ \\
\hline $\begin{array}{l}\text { Código regional } \\
{[\mathrm{O}][\mathrm{R}]}\end{array}$ & 3.19 .6 & $\begin{array}{l}\text { O código de classificação que indica a codificação de vídeo e o } \\
\text { hardware regional necessário para realizar o jogo (por exemplo, a } \\
\text { América do Norte NTSC-U/C, o Japão e a Ásia NTSC-J, a Europa, a } \\
\text { Nova Zelândia e a Austrália PAL). }\end{array}$ \\
\hline Idioma [R] & 6.11 & A(s) língua(s) em que o jogo transmite informações. \\
\hline $\begin{array}{l}\text { Data de } \\
\text { lançamento } \\
{[\mathrm{O}][\mathrm{R}]}\end{array}$ & 2.8 & A data do lançamento público/comercial do jogo. \\
\hline Faixa Etária [R] & 7.7 & $\begin{array}{l}\text { A classificação da faixa etária atribuída pelo tipo do conteúdo } \\
\text { apresentado pelos jogos, fornecida por organizações como associações } \\
\text { profissionais, distribuidores de jogos ou criadores. Alguns jogos mais } \\
\text { antigos não têm essa informação de classificação. (por exemplo, God } \\
\text { of war: Ascension: ESRB; M (Mature 17+): Sangue excessivo, } \\
\text { violência intensa e linguagem inapropriada). }\end{array}$ \\
\hline $\begin{array}{l}\text { Website oficial } \\
{[\mathrm{R}]}\end{array}$ & 4.6 & $\begin{array}{l}\text { Um Uniform Resource Locator (URL) do site para o jogo ou das } \\
\text { empresas oficialmente associadas ao jogo. }\end{array}$ \\
\hline Prêmio $[R]$ & 7.28 & $\begin{array}{l}\text { O nome de todos os prêmios que o jogo ganhou e o órgão concedente } \\
\text { que os premiou. }\end{array}$ \\
\hline $\begin{array}{l}\text { Opções de } \\
\text { personalização }\end{array}$ & $\begin{array}{l}\text { Não há regra } \\
\text { associada }\end{array}$ & $\begin{array}{l}\text { As opções no jogo para o nível de dificuldade e caracteres que podem } \\
\text { ser modificados pelo jogador para a experiência personalizada. }\end{array}$ \\
\hline Embalagem & 3.4 & $\begin{array}{l}\text { Todos os itens incluídos na embalagem original do jogo (por exemplo, } \\
\text { dois discos de jogos, manual). }\end{array}$ \\
\hline Gênero $[\mathrm{O}][\mathrm{R}]$ & 6.3 & $\begin{array}{l}\text { A natureza geral de um jogo com base em seus objetivos, tipos de } \\
\text { regras, características distintivas, modos de ação, modos de } \\
\text { jogabilidade e como um jogador interage com ele. }\end{array}$ \\
\hline Resumo & 7.10 & $\begin{array}{l}\text { Uma breve declaração dos eventos subjacentes que compõem a } \\
\text { história do jogo. }\end{array}$ \\
\hline $\begin{array}{l}\text { Tempo estimado } \\
\text { de conclusão }\end{array}$ & 7.22 & O tempo médio estimado para completar o jogo. \\
\hline Tipos de finais & $\begin{array}{l}\text { Não há regra } \\
\text { associada }\end{array}$ & $\begin{array}{l}\text { As características que descrevem como o jogo termina e/ou conteúdo } \\
\text { pós-jogo. }\end{array}$ \\
\hline Estilo visual $[\mathrm{R}]$ & $\begin{array}{l}\text { Não há regra } \\
\text { associada }\end{array}$ & $\begin{array}{l}\text { A aparência visual predominante e reconhecível do jogo (por exemplo: } \\
\text { histórias em quadrinhos, pixel art, Lego). }\end{array}$ \\
\hline Dimensão & 3.17 .2 & $\begin{array}{l}\text { A percepção pretendida da profundidade das entidades representadas } \\
\text { dentro do jogo (por exemplo, 2D, 3D). }\end{array}$ \\
\hline Pontos de vista & $\begin{array}{l}\text { Não há regra } \\
\text { associada }\end{array}$ & $\begin{array}{l}\text { Perspectiva a partir da qual o jogador experimenta a jogabilidade (por } \\
\text { exemplo, primeira pessoa, terceira pessoa). }\end{array}$ \\
\hline Screenshots [R] & $\mathrm{J} 3.5$ & Imagens captadas durante a execução do jogo. \\
\hline Trailers $[\mathrm{R}]$ & $\mathrm{J} 3.5$ & $\begin{array}{l}\text { Vídeos lançados pelo desenvolvedor/editor do jogo para fins } \\
\text { promocionais. }\end{array}$ \\
\hline $\begin{array}{l}\text { Vídeos de } \\
\text { Gameplay }[\mathrm{R}]\end{array}$ & $\mathrm{J} 3.5$ & $\begin{array}{l}\text { Vídeo da jogabilidade excluindo coisas como apresentações, cutscenes } \\
\text { ou trailers. }\end{array}$ \\
\hline Nota & 5.9 & Todas as outras características notáveis do jogo. \\
\hline
\end{tabular}

Fonte: Traduzido e adaptado de Lee; Clarke e Perti (2015). 
Com base nesses metadados, serão apresentados a seguir os elementos que devem conter em um registro catalográfico além do tipo de conteúdo, tipo de mídia e tipo de suporte. É importante ressaltar que o RDA apenas instrui quais elementos são essenciais para o registro e não como apresentá-los. Sendo assim, os itens foram dispostos conforme estabelecido pelo AACR2.

\subsection{TÍTULO E INDICAÇÃO DE RESPONSABILIDADE}

Como os jogos são, geralmente, trabalhos colaborativos, o título será o ponto de acesso principal (RDA 6.27.1.3). Como os jogos eletrônicos são considerados imagens em movimento, a fonte preferida de informação para o registro do título é a tela de título do jogo (RDA 2.2.2.3). Caso não possua o equipamento necessário para a visualização do título, pode-se usar um rótulo "impresso ou afixado no recurso" (RDA 2.2.2.3.1). Se o título não aparecer no recurso, ele pode ser retirado do material adicional, descrição publicada, um rótulo que não é emitido com o próprio recurso ou outra fonte (RDA 2.2.4), registrando entre colchetes. Sempre que o título é tirado de uma fonte diferente da tela de título, uma nota deve ser fornecida para identificar a fonte do título (2.17.2.3).

Palavras introdutórias que claramente não fazem parte do título não devem ser transcritas (RDA 2.3.1.6). Em casos em que o título inclui um nome que normalmente seria tratado como parte de uma declaração de responsabilidade, ou como o nome de um editor, distribuidor, etc., se considerado parte integrante do título, deve ser incluído no registro (RDA 2.3.1.5).

Para os títulos de jogos que fazem parte de uma franquia, como não há uma definição clara do que é, sugere-se registrar a franquia como parte do título. Caso seja necessária uma distinção, registrar a franquia em notas.

Se o recurso for uma compilação de mais de um jogo, registre o título coletivo quando possível (RDA 2.3.2.6.1). Registre os nomes dos jogos individuais como pontos de acesso secundários, se considerado importante para identificação ou acesso. Se não houver título coletivo, registre os títulos na ordem em que aparecem no recurso (RDA 2.3.2.9).

Um título original em um idioma diferente do título apresentado no recurso deve ser tratado como um título paralelo se for apresentado como o equivalente a um título próprio (RDA 2.3.3.1). Diferentemente da AACR2, o título paralelo não precisa vir da mesma fonte que o título propriamente dito, mas pode vir de qualquer fonte dentro do recurso (RDA 2.3.3.2). As formas variantes do título, se considerado importante, devem ser registradas. Elas podem vir de qualquer fonte, incluindo variantes transcritas do recurso (RDA 2.3.6.2).

A indicação de responsabilidade de um jogo eletrônico, por ser um trabalho colaborativo, normalmente é feita para uma entidade coletiva. O nome da entidade coletiva deve ser registrado tal como aparece no jogo (RDA 11.2.2.4). A informação deve, preferivelmente, ser retirada da mesma fonte que o título. Caso não seja 
possível, pode ser retirado de outra fonte dentro do recurso (RDA 2.4.2.2). Outras formas do nome podem ser transcritas como nomes variantes (RDA 11.2.2.5).

Para o registro de corpo corporativo, os termos adjetivos ou abreviatura indicando incorporação (por exemplo, Inc., Ltda.), termos que indicam a propriedade estatal de um órgão corporativo e palavras ou frases, abreviadas ou total, indicando o tipo de entidade devem ser omitidos. A menos que sejam necessários para deixar claro que é o nome de uma corporação (RDA 11.2.2.10).

Como o ponto de acesso principal para os jogos é o título, os pontos de acesso secundários são as indicações de responsabilidade. Não é necessário fazer pontos de acesso secundários para todos os nomes registrados na indicação de responsabilidade ou em qualquer campo de crédito.

\subsection{EDIÇÃO}

A edição deve ser transcrita conforme aparece na fonte de informações (RDA 2.5.1.4). Diferentemente da AACR2, a palavra "edição" não é abreviada a menos que seja abreviada na fonte. A designação deve ser, preferencialmente, retirada da mesma fonte do título (RDA 2.5.2.2).

Se a designação de edição fizer parte do título, informações complementares do título ou menção de responsabilidade, então deve ser registrada como parte integrante do elemento ao qual está integrada ou vinculada. Não se deve registrar novamente como uma designação de edição (RDA 2.5.2.6).

Para um recurso que não possua uma menção de edição, mas contém mudanças significativas de outras edições, pode-se fornecer uma declaração de edição se for considerada importante para identificação ou acesso. Apenas deve-se indicar que a informação foi tirada de uma fonte externa ao recurso (RDA 2.5.1.4 adição opcional). Assim, qualquer indicação de plataforma ou sistema que possa ser interpretado como uma indicação de edição pode ser transcrita como tal, desde que entre colchetes.

\subsection{DADOS DE PUBLICAÇÃO}

Os dados de publicação é uma menção que identifica o(s) lugar(es) de publicação, o(s) editor(es) e a(s) data(s) de publicação de um recurso.

O local de publicação deve ser transcrito conforme aparece na fonte de informação (RDA 2.8.1.4), incluindo o nome do local (cidade) e a jurisdição ou jurisdições principais (estado, província, país) se presente na fonte de informação (RDA 2.8.2.3). As informações devem ser retiradas, preferencialmente, da mesma fonte do nome do editor. Se mais de um local de publicação for indicado no recurso, os nomes dos locais devem ser transcritos na ordem indicada pela sequência, layout ou tipografia dos nomes na fonte de informação (RDA 2.8.2.4). Caso nenhum local de publicação for identificado, deve ser fornecido um local de publicação ou local de publicação provável se puder ser determinado, entre colchetes (RDA 2.8.2.6). A expressão "Lugar de publicação não identificado" tem que ser registrada se não for determinado um local ou jurisdição de publicação conhecido ou provável (RDA 2.8.2.6.5). 
Quanto ao editor, a informação deve ser retirada, preferencialmente, da mesma fonte que o título (RDA 2.8.4.2). A transcrição do nome do editor deve ser feita na forma como aparece na fonte de informação (RDA 2.8.1.4). Se os níveis de uma hierarquia corporativa não são necessários para identificar o editor, podem ser omitidos sem nenhuma indicação de tal omissão (RDA 2.8.4.3 omissão opcional).

Se houver mais de um editor, os nomes dos editores devem ser registrados na ordem indicada pela sequência, layout ou tipografia dos nomes na fonte de informação (RDA 2.8.4.5). Caso não haja editor, registra-se o distribuidor ou fabricante se identificados. Registra-se a expressão "Editor não identificado", caso nenhum editor for identificado no recurso ou em outras fontes (RDA 2.8.4.7).

A data de publicação, do mesmo modo que o editor tem as mesmas fontes (RDA 2.7.6.2) e deve ser transcrita conforme aparece na fonte de informação (RDA 2.7.6.3). Se nenhuma data for identificada, uma data retirada de uma fonte externa ou a data aproximada de publicação deve ser registrada (RDA 2.8.6.6). O RDA não restringe a data apenas ao ano, possibilitando o registro completo da data se indicado no recurso.

A data de direitos autorais, quando registrada, deve ser precedida do símbolo de direitos autorais $\odot$ ou o símbolo de copyright de fonograma ( pode ser retirada de qualquer fonte (RDA 2.11.1.2).

\subsection{DESCRIÇÃO FÍSICA}

A descrição física do suporte ou suportes deve ser baseada em evidências apresentadas pelo próprio jogo ou em qualquer material ou recipiente que o acompanha. Se informações adicionais forem consideradas importantes para identificação ou seleção, podem ser obtidas de qualquer fonte (RDA 3.1.1).

Para a descrição física, os dados usados dependerão da natureza e do objetivo da descrição. O registro pode conter apenas o tipo de mídia, a extensão e outras características de cada suporte; ou o tipo de mídia predominante e extensão em termos gerais; ou registrar o tipo de mídia e a extensão de cada mídia (RDA 3.1.4).

O registro da extensão de um jogo deve conter o número de unidades e o tipo de suporte (RDA 3.4.1.3). Se o número exato de unidades não puder ser facilmente determinado, pode ser feito o registro de um número aproximado precedido pela palavra "aproximadamente" (RDA 3.4.1.4). O mesmo pode ser para o número de subunidades em cada unidade (RDA 3.4.1.9).

Ao descrever uma coleção como um todo, a extensão dependerá, também, da natureza da coleção e o propósito da descrição. Pode ser registrado apenas o número de itens, contêineres ou volumes; ou o espaço de armazenamento; ou o número e tipo de unidade (RDA 3.4.1.11).

As dimensões devem ser registradas em centímetros, conforme o suporte. Por exemplo, o registro de um disco é feito a partir de seu diâmetro (RDA 3.5.1.4.4). Para cartuchos de computador, o comprimento do lado do cartucho que deve ser inserido na máquina é a dimensão utilizada para o registro (RDA 3.5.1.4.2).

$\mathrm{O}$ registro deve utilizar o símbolo métrico $\mathrm{cm}$ e as dimensões precisam ser arredondadas para o 
próximo centímetro inteiro (por exemplo, se a altura média 17,2 centímetros, gravar $18 \mathrm{~cm}$ ) (RDA 3.5.1.3). O sistema métrico para registrar as dimensões poderá ser outro, se preferir (RDA 3.5.1.3 alternativa). As dimensões podem incluir medidas de altura, largura, profundidade, comprimento, calibre e diâmetro (RDA 3.5.1.1).

Se o jogo estiver em um recipiente, pode ser feito o registro da altura, largura e profundidade do recipiente, caso seja considerado importante para identificação ou seleção. As dimensões do recipiente podem ser as únicas a serem registradas (RDA 3.5.1.5).

Caso o jogo possua mais de um suporte, e todos os suportes são do mesmo tipo e tamanho, basta registrar as dimensões de um único suporte. Se os suportes são do mesmo tipo, mas diferem em tamanho, as dimensões devem ser registradas do menor para o maior ou vice-versa (RDA 3.5.1.6). Isso é equivalente para o recipiente (RDA 3.5.1.7).

Como os jogos eletrônicos não consistem principalmente em som gravado é necessário registrar a presença ou ausência de som. Embora a maioria dos jogos possua algum efeito sonoro, alguns podem não possuir. O termo utilizado para o registro pode ser sonoro ou silencioso (RDA 7.18.1.3).

As cores presentes no conteúdo de um jogo também devem ser registradas. As cores preta, branca e tons de cinza são considerados cores únicas. Sugere-se adotar os termos já conhecidos, "preto e branco" ou "colorido" (RDA 7.17.3.3 alternativa). Para jogos que utilizam tons de uma única cor, desde que não seja cinza, o termo sugerido é "monocromático" (RDA 7.17.3.3). Se considerados importantes, outros detalhes do conteúdo de cor podem ser registrados como, por exemplo, a tonalidade da cor de jogos monocromáticos (monocromático em tons de vermelho) (RDA 7.17.1.4).

Para jogos de computador, smartphones e jogos on-line, sugere-se registrar o tamanho de arquivo caso possa ser facilmente determinado. $\mathrm{O}$ registro pode ser feito em bytes, kilobytes (KB), megabytes (MB) ou gigabytes (GB), conforme apropriado (RDA 3.19.4.3).

\subsection{SÉRIE}

O registro do título próprio da série deve ser feito conforme aparece na fonte de informações (RDA 2.12.1.4). Se o jogo pertence a mais de uma série ou subséries, cada declaração da série tem que ser registrada separadamente (RDA 2.12.1.5).

A numeração de uma série deve ser registrada, aplicando as diretrizes gerais para números expressos como numerais ou como palavras (RDA 2.12.9.3). Se a numeração aparecer na fonte de informações em mais de uma forma, o registro deverá ser feito com a numeração que está na forma do título próprio da série. Se esse critério não se aplica, a numeração que aparece primeiro deve ser transcrita (RDA 2.12.9.5).

Quando a numeração é gramaticalmente integrada com o título da série, deve ser transcrita como parte do título próprio da série. Se o jogo descrito consistir em mais de uma parte e a numeração for diferente entre as partes, a numeração deve ser omitida (RDA 2.12.2.3). 
Se uma frase como "Nova série", "Segunda série", etc., aparecer com uma série não numerada na fonte de informação, a frase deve ser transcrita como um título de uma subsérie. Caso a série seja numerada, então se registra a frase como parte da numeração das séries (RDA 2.12.10.4).

As instruções sobre a gravação de títulos próprios das subséries (RDA 2.12.10.3) e de sua numeração (RDA 2.12.17.3) são as mesmas para séries.

\subsection{NOTAS}

Se consideradas importantes para esclarecimentos ou justificativas, o catalogador pode atribuir notas sobre as instruções específicas aplicadas na criação do ponto de acesso autorizado; notas que justificam a escolha de um título de trabalho preferido; notas que limitam o uso do ponto de acesso e notas diferenciando trabalhos com títulos similares. (RDA 5.9.1.3).

Elementos como gênero (RDA 6.3.1.3); requisitos do equipamento ou sistema (RDA 3.20.1.3); URL (RDA 4.6.1.3); idioma (RDA 6.11.1.3); dublador, narrador e/ou apresentador (RDA 7.23.1); produção artística e/ou técnica (RDA 7.24); premiação (RDA 7.28.1.3); público (RDA 7.7.1.3); resumo (RDA 7.10.1.3) e tempo estimado de duração do jogo (RDA 7.22.1.3) possuem regras próprias para a sua descrição.

Outros elementos que não são abordados no RDA e são considerados importantes para descrever adequadamente os jogos de forma reconhecível pelos usuários podem ser registrados em notas também. Entre esses elementos, pode-se destacar: recursos de rede, conectividade, número de jogadores, opções de personalização, screenshots vídeos de gameplay, entre outros.

\subsection{NÚMERO NORMALIZADO}

Número normalizado é constituído de um grupo de caracteres que servem como identificadores de um recurso. Se aplicável, o catalogador deve registrar um identificador internacionalmente reconhecido (RDA 2.15). O número normalizado pode ser retirado de qualquer fonte (RDA 2.15.1.2). O registro deve ser precedido com um nome comercial ou o nome da agência, etc., responsável por atribuir o identificador, se for facilmente verificável (RDA 2.15.1.4).

Para jogos compostos por mais de uma parte, o número adotado deve representar o recurso como um todo (RDA 2.15.1.5). Se houver mais de um identificador, os identificadores devem ser registrados separados entre si por um hífen. Para três ou mais identificadores, apenas o primeiro e o último identificados precisam ser registrados. Neste caso, devem ser separados por uma barra diagonal (RDA 2.15.1.5 alternativa).

\subsection{EXEMPLOS DE REGISTROS DE JOGO ELETRÔNICO}

A seguir, são apresentados e descritos três registros de jogos em formato Machine Readable Cataloguing (MARC 21), com a descrição em RDA. O formato MARC consiste em um padrão de metadados legível por computador (MEY; SILVEIRA, 2009). A escolha dos títulos deve-se por sua popularidade e por representar os principais suportes dos jogos eletrônicos. 
Quadro 2 - Exemplo de registro de um cartucho para computador (Super Mario World)

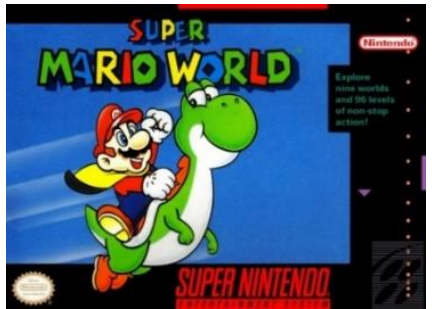

Frente da caixa

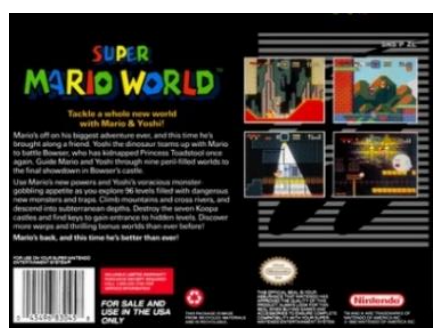

Verso da caixa

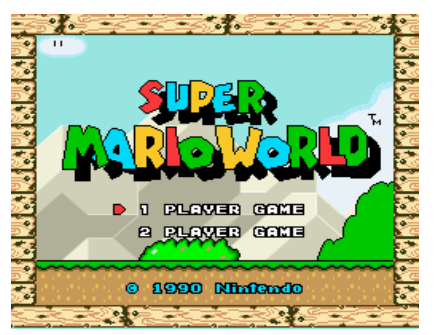

Tela inicial

24500 \$a Super Mario World / \$c Nintendo. -

250 \$a [Super Nintendo].

260 \$a [Redmond, WA] : \$b [Nintendo of America Inc.], \$c @1990

300

336

336

337

338

344

380

500

\$a 1 cartucho: \$b sonoro, colorido; \$c $14 \mathrm{~cm} \$$ f em caixa $\$ \mathrm{~g} 17$ x 24 x $4 \mathrm{~cm}$

$\$$ a imagem em movimento bidimensional $\$ 2$ rdacontent

\$a programa de computador \$2 rdacontent

$\$$ a computador \$a rdamedia

$\$$ a cartucho para computador $\$ 2$ rdacarrier

$\$$ a digital $\$ 2$ rda

\$a Jogo de plataforma.

\$a Para dois jogadores.

\$a Direção de Takashi Tezuka; produzido por Shigeru miyamoto.

\$a Mario e seu dinossauro, Yoshi, devem atravessar sete mundos cheios de perigo para combater o Bowser e resgatar a Princesa Toadstool mais uma vez.

538 \$a Requisitos do sistema: Super Nintendo Entertainment System.

546 \$a Idioma em inglês.

$630 \quad 00 \quad$ \$a Mario \$v Jogos eletrônicos.

$650 \quad 04 \quad$ \$a Jogos de plataforma.

$650 \quad 04 \quad \$ a$ Videogames Nintendo.

$700 \quad 1$ \# \$a Tezuka, Takashi \$e diretor

$700 \quad 1$ \# \$a Miyamoto, Shigeru, \$d 1952- \$e produtor

$710 \quad 2$ \# \$a Nintendo (Firm), \$e editora.

753 \$a Super Nintendo Entertainment System

$787 \quad 1$ \# $\$$ \$a Donkey Kong

787 1 \# \$a Super Mario World 2 : Yoshi’s Island

787 1 \# \$a Mario Kart

$787 \quad 1$ \# $\quad$ \$a Super Smash Bros.

Fonte: Elaborado pelos autores (2017).

O primeiro registro trata-se de um clássico da Nintendo, o "Super Mario World". Desenvolvido em 1990, o jogo foi lançado em diversas plataformas. Sendo assim, como não há uma indicação de edição, podese indicar a plataforma no campo 250. A criação de ponto de acesso secundário para plataforma também pode auxiliar na recuperação dos jogos eletrônicos, sendo assim, a plataforma deve ser registrada no campo específico para isso, o campo 753 do MARC.

Além de descrever o recurso de forma mais detalhada, o RDA apresenta outra vantagem em relação ao AACR2, a possibilidade de incluir ligações com outras obras, expressões, pessoas e entidades. O personagem principal do jogo descrito, por exemplo, surgiu em outro jogo chamado Donkey Kong (JETT et al, 2016). O jogo também possui sequências como Super Mario World 2: Yoshi's Island, spin-offs como o 
popular Mario Kart e jogos de cross-over como o Super Smash Bros. Esta relação entre as obras é indicada no campo 787 do MARC.

Quadro 3 - Exemplo de registro de um disco para computador (Crash Bandicoot)

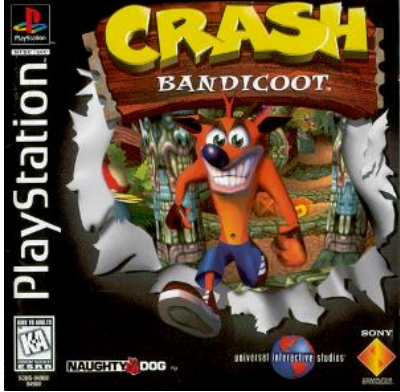

Frente da capa

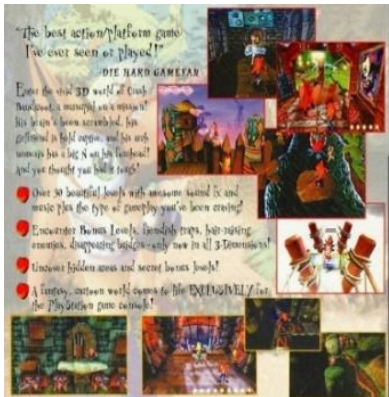

Verso da capa

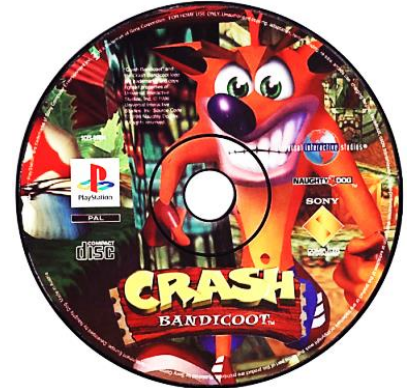

Suporte

$245 \quad 00 \quad$ \$a Crash Bandicoot / \$c Naughty Dog. -

$250 \quad$ \$a [PlayStation]

260 \$a [Foster City, CA] : \$b Universal Interactive Studios, Sony Computer Entertainment, \$c (C)1996

300 \$a 1 disco de computador: \$b sonoro, colorido; \$c 43/4 pol.

336 \$a imagem em movimento tridimensional \$2 rdacontent

336 \$a programa de computador \$2 rdacontent

$337 \quad$ \$a computador \$a rdamedia

338 \$a disco de computador \$2 rdacarrier

$344 \quad \$$ a digital \$2 rda

$345 \quad$ \$a 3D

346

380

\$a Jogo de plataforma em 3D.

\$a Título do rótulo do disco.

\$a Para um jogador.

\$a Ajude o marsupial Crash Bandicoot a resgatar sua namorada que é mantida em cativeiro pelo seu inimigo, Neo Cortex.

\$a Classificação ESRB: K-A, crianças e adultos.

$538 \quad$ \$a Requisitos do sistema: PlayStation.

546 \$a Idioma em inglês.

$630 \quad 00 \quad$ \$a Crash \$v Jogos eletrônicos.

$650 \quad 04 \quad$ \$a Jogos de plataforma.

$650 \quad 04$ \$a Jogos eletrônicos.

$710 \quad 2 \# \quad$ \$a Naughty Dog, Inc. \$e editora.

710 2\# \$a Universal-Internacional (Firm). \$e distribuidora

710 2 \# \$a Sony Computer Entertainment. \$e distribuidora

753 \$a PlayStation

Fonte: Elaborado pelos autores (2017).

O segundo jogo, diferentemente do primeiro, é um jogo em 3D. Lançado pela Naughty Dog, o jogo é nomeado com o nome de seu personagem principal, Crash Bandicoot. Assim como o primeiro registro, o jogo também foi lançado para outras plataformas e como não há indicação de edição, pode-se indicar a plataforma PlayStation. Além disso, o jogo apresenta indicação de editora e mais de uma indicação de distribuidora. Não é obrigatório registrar todas as distribuidoras e criar pontos de acesso secundário para tal. Neste caso em 
específico, como a empresa Sony é responsável pelo lançamento do console PlayStation, é recomendável a criação do ponto de acesso secundário, visando a recuperação por parte do usuário.

Quadro 4 - Exemplo de registro de um recurso on-line (Angry Birds)

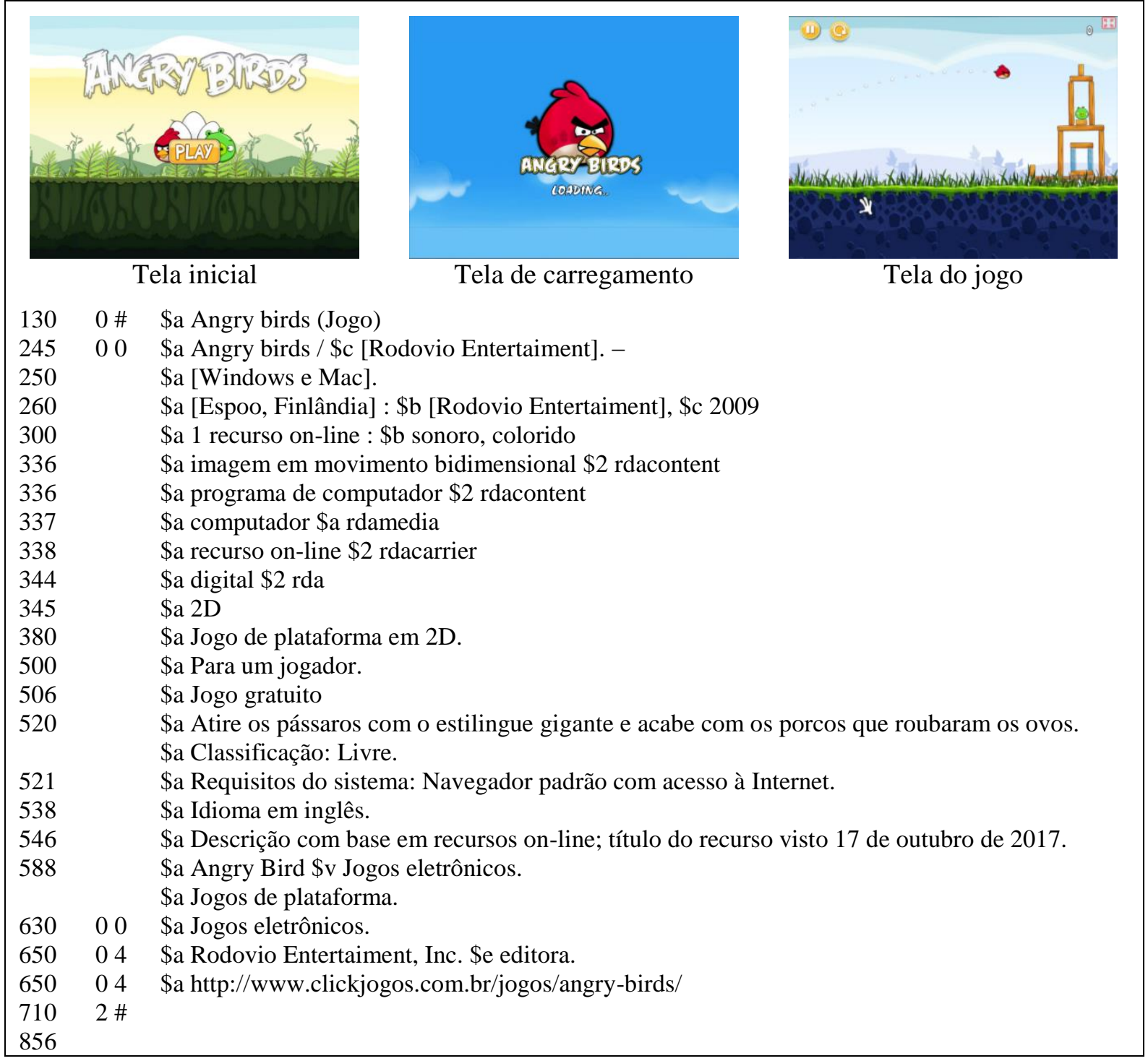

Fonte: Elaborado pelos autores (2017).

Lançado inicialmente para smartphones, o jogo Angry Birds está disponível on-line. O URL pode ser registrado no campo 856 do MARC. Como o jogo não possui muitas informações no próprio suporte para a descrição, requer a busca em outras fontes. O título do jogo também é o título da franquia. Caso seja identificada demanda por maior esclarecimento, pode-se elaborar uma nota com essa finalidade.

\section{CONSIDERAÇÕES FINAIS}

Como os jogos eletrônicos possuem particularidades únicas e diferentes interpretações por parte dos usuários, nem sempre se encaixam nos modelos existentes de registro que tentam descrever informações 
relevantes e as relações entre elas. Sendo assim, o propósito dessa pesquisa foi analisar as regras aplicáveis pelo RDA para catalogação de jogos eletrônicos.

Para tanto, foram definidos jogos eletrônicos e conceitos relacionados, identificando elementos necessários para catalogar jogos eletrônicos. Para tanto, utilizou-se um esquema de metadados padronizado para jogos eletrônicos e o vocabulário controlado desenvolvido pelo grupo GAMER em parceria com o SIMM. Destaca-se que foram apresentados, neste estudo, os elementos considerados essenciais para a descrição de jogos eletrônicos, porém o RDA recomenda outros dados que podem ser usados para descrição, dependendo da política de catalogação da biblioteca.

Uma das principais dificuldades encontradas para o tratamento dos jogos eletrônicos é o registro de seus metadados. Apesar de ser necessário que o bibliotecário tenha conhecimento da terminologia usada nos jogos, o problema está em como representar essas informações. Mesmo no RDA, que trouxe mais subsídios para a descrição de jogos eletrônicos, há questões que as regras existentes não conseguem esclarecer. Isso ressalta a necessidade de criar um grupo bem estabelecido de metadados para solucionar estas questões, visando uma representação que atenda às demandas dos usuários.

Outro fator que pode desfavorecer o trabalho do catalogador é a falta de um dispositivo de reprodução para os jogos, principalmente os mais antigos. Sem um aparelho de reprodução, as informações deverão ser retiradas de fontes externas ao recurso. Essa tarefa pode ser onerosa para um bibliotecário, pois a procura pelas informações pode envolver maior exigência de tempo. Sites destinados a jogos como Steam, Moby Games ou TheGamesDB.net podem auxiliar na coleta das informações pertinentes para a descrição.

É importante salientar que atualmente a aplicação do RDA, mesmo com todas as possibilidades que o formato MARC apresenta, é prejudicada. Mesmo com a criação de novos campos como o 336 para tipo de conteúdo, 337 para tipo de mídia e 338 para o tipo de suporte, os conceitos do RDA não são totalmente descritos em um registro bibliográfico elaborado no formato MARC 21.

Com base nisso, é possível concluir que o catalogador precisa de mais conhecimento a respeito do que está catalogando e tempo para realizar uma pesquisa mais ampla em outras fontes de informação. Desse modo, os registros poderiam ser elaborados com mais dados e mais bem estruturados.

Para finalizar, sugere-se como pesquisas futuras um maior aprofundamento sobre os conceitos envolvidos acerca do RDA e sobre as suas funcionalidades para a catalogação de jogos eletrônicos, bem como de outros recursos relacionados. Recomenda-se, ainda, o desenvolvimento de pesquisas empíricas que demonstrem os problemas e vantagens do uso do RDA para a descrição de jogos eletrônicos, possibilitando a criação de uma representação consistente que reflita em possíveis melhorias na recuperação da informação, atendendo os usuários finais. 


\section{REFERÊNCIAS}

ARANHA, Gláucio. O processo de consolidação dos jogos eletrônicos como instrumento de comunicação e de construção de conhecimento. Cien. Cogn., Rio de Janeiro, v. 3, n. 1, p. 10-20, nov. 2004. Disponível em: http://www.cienciasecognicao.org/revista/index.php/cec/article/view/473. Acesso em: 28 abr. 2017.

BAPTISTA, Dulce. Catalogação: uma revolução em curso. In: ENCONTRO NACIONAL DE PESQUISA EM CIÊNCIA DA INFORMAÇÃO, 9., 2008, São Paulo. Anais eletrônicos [...] São Paulo: ANCIB, p. 1-9. Disponível em: http://www.enancib2008.com.br/. Acesso em 18 abr. 2017.

CERVO, Amado Luiz; BERVIAN, Pedro Aicino; SILVA, Roberto da. Metodologia científica. 6. ed. São Paulo: Pearson, 2007.

GROAT, Greta de. A History of Video Game Cataloging in U.S. Libraries. Cat Classif Q, v. 53, n. 2, p. 135-156, 2015. Disponível em: http://dx.doi.org/10.1080/01639374.2014.954297. Acesso em: 24 jun. 2017.

JETT, Jacob et al. A conceptual model for video games and interactive media. J Assoc. Inf. Sci. Technol., v. 63, n. 3, p. 505-517, mar. 2016. Disponível em: https://doi.org/10.1002/asi.23409. Acesso em: 14 nov. 2017.

LEE, Jin Ha; CLARKE, Rachel Ivy; PERTI, Andrew. Empirical Evaluation of Metadata for Video Games and Interactive Media. J. Assoc. Inf. Sci. Technol., v. 66, n. 12, p. 2609-2625, 2015. Disponível em: https://doi.org/10.1002/asi.23357. Acesso em: 28 out. 2017.

MACHADO, Raquel Bernadete; PEREIRA, Ana Maria. Aspectos da catalogação e do RDA: contribuições teóricas da literatura nacional e internacional. Enc. Bibli: R. Eletr. Bibliotecon. Ci. Inf., v. 22, n.49, p. 89101, maio/ago., 2017. Disponível em: https://periodicos.ufsc.br/index.php/eb/article/view/1518-

2924.2017v22n49p89. Acesso em: 28 out. 2017.

MEY, Eliane Serrão Alves; SILVEIRA, Naira Christofoletti. Catalogação no plural. Brasília: Briquet de Lemos/Livros, 2009.

OLIVER, Chris. Introdução à RDA: um guia básico. Tradução de Antônio Agenor Briquet de Lemos. Brasília, DF: Briquet de Lemos/Livros, 2011.

OWENS, Trevor. Yes, the Library of Congress has video games: An interview with David Gibson. The Signal. [S.l.], 26 set. 2012. Disponível em: https://blogs.loc.gov/thesignal/2012/09/yes-the-library-ofcongress-has-video-games-an-interview-with-david-gibson/. Acesso em 23 jun. 2017.

PICCO, Paola. El objeto de la catalogación en el marco de las FRBR y el nuevo código de catalogación. Enc. Bibli: R. Eletr. Bibliotecon. Ci. Inf., Florianópolis, v. 14, n. 28, p. 150-162, 2009. Disponível em: https://periodicos.ufsc.br/index.php/eb/article/view/1518-2924.2009v14n28p150. Acesso em: 23 jun. 2017.

RDA TOOLKIT: Resource Description \& Access. [S.l.], 2010. Disponível em: http://access.rdatoolkit.org/. Acesso em: 20 out. 2017.

SANTOS JÚNIOR, Roberto Lopes dos; NUNES, Vanderson Monteiro. Estudo da preservação digital dos videogames sob o viés da arquivologia. Inf. \& Soc.: Est., João Pessoa, v.26, n.3, p. 31-46, set./dez. 2016. Disponível em: http://periodicos.ufpb.br/ojs/index.php/ies/article/view/29322/16867. Acesso em: 3 maio 2017. 
SANTOS, Plácida Leopoldina Ventura Amorim da Costa; CORRÊA, Rosa Maria Rodrigues. Catalogação: trajetória para um código internacional. Niterói: Intertexto, 2009.

SILVA, Eliana Barboza de Oliveira et al. Conceituação e aplicação do novo padrão para descrição bibliográfica Resource Description and Access (RDA). CRB-8 Digital, São Paulo, v. 5, n. 1, p. 113-123, jan. 2012. Disponível em: http://revista.crb8.org.br/index.php/crb8digital/article/viewFile/74/76. Acesso em: 5 out 2017.

TILLETT, Barbara. Visão geral do esboço da Declaração dos Princípios de Catalogação. [S.l. : s.n.], 2007. Disponível em:

http://www.imeicc5.com/download/portuguese/Presentations4_Cataloguing\%20principlesIME\%20ICC\%20overview_BT_Port.pdf. Acesso em 15 jun. 2017. 